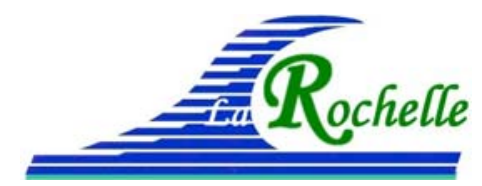

XVèmes Journées Nationales Génie Côtier - Génie Civil

La Rochelle, 29 au 31 mai 2018

DOI:10.5150/jngcgc.2018.096 ～(C) Editions Paralia CFL

disponible en ligne - http://www.paralia.fr - available online

\title{
Stratégies de modélisation des phénomènes de submersion par franchissement de paquets de mer
}

\author{
Alexandre NICOLAE LERMA ${ }^{1}$, Rodrigo PEDREROS ${ }^{2}$, \\ Thomas BULTEAU ${ }^{1}$, Yann BALOUIN ${ }^{3}$
}

1. BRGM, Parc Technologique Europarc 24 Avenue Léonard de Vinci 33600 Pessac, France.a.nicolaelerma@brgm.fr ; t.bulteau@brgm.fr

2. BRGM, Direction Risques et Prévention, 3 Avenue Claude Guillemin, 4500 Orléans, France.r.pedreros@brgm.fr

3. BRGM, Direction Régionale Occitanie, 1039 rue de Pinville, 34000 Montpellier, France.y.balouin@brgm.fr

\section{Résumé :}

La prise en compte de l'aléa de submersion sur les sites soumis conjointement à l'aléa de submersion par débordement et par franchissement de paquets de mer représente un défi important. En effet, si les modèles et les méthodes permettent aujourd'hui de reproduire de manière satisfaisante les phénomènes de submersion marine par débordement, y compris en milieu urbain, la reproduction des phénomènes de franchissement par paquet de mer fait appel à des modèles dont le degré de maturité est nettement inférieur. Une difficulté supplémentaire réside dans la validation des performances des modèles en configuration réelle, les observations de terrain étant le plus souvent partielles ou lacunaires.

L'objectif de cette contribution est de réaliser une étude comparative des stratégies de modélisation en $2 \mathrm{DV}$ et 3D mises en place sur le site du lido de la lagune de Leucate afin de reproduire les phénomènes de submersion marine par franchissement. Les hypothèses et les approches envisagées sont comparées au travers de la reproduction d'un évènement historique bien documenté et d'un événement de période de retour centennal.

Mots-clés : Alea submersion, SWASH, Modélisation numérique, Front de mer, Contexte microtidal.

\section{Introduction}

Ces dernières années, la caractérisation de l'aléa de submersion marine s'est considérablement développée sur les littoraux français. Associé à ce besoin de mieux décrire l'impact des tempêtes, les méthodes évoluent, en fonction des caractéristiques particulières des sites étudiés (exposition, aménagements de protection, sensibilité à la hausse du niveau de la mer...), mais également grâce aux gains en maturité des outils de modélisation numérique. Les études de submersion couvrent un spectre large allant d'études basées sur des approches SIG répondant par exemple aux directives TRI 


\section{Thème 7 - Risques côtiers}

jusqu'à la mise en place de plateforme de prévision de l'aléa en fonction des prévisions météo marines (LECACHEUX et al., 2016).

La modélisation de la submersion par débordement est aujourd'hui relativement maitrisée. Toutefois, l'aléa de submersion est souvent causé par des franchissements (LE ROY et al., 2015 ; GALLIEN, 2016 , NICOLAE LERMA et al., 2018). Or, la modélisation des franchissements en conditions réelles se confronte à plusieurs défís majeurs. Elle exige de travailler à une résolution fine, de l'ordre du mètre pour représenter correctement le comportement des vagues et leur interaction avec le front de mer. En effet, l'ampleur des franchissements est fortement dépendante de la morphologie de la plage et du haut de plage. Une difficulté supplémentaire est la validation de ces méthodes et de leurs résultats (NICOLAE LERMA et al., 2013 ; GALLIEN, 2016), les observations de terrain permettant de valider les modélisations de volumes franchissants étant le plus souvent imprécises et uniquement qualitatives.

Le travail réalisé ici consiste à démontrer la nécessité de développer des modélisations des franchissements à haute résolution afin d'évaluer de manière pertinente la sensibilité des fronts de mer des littoraux microtidaux, qui ont la particularité d'une urbanisation en front de mer confrontée directement aux franchissements de paquets de mer. Pour cela, les résultats obtenus en matière de submersion à partir de différentes approches méthodologiques sont comparés et analysés, notamment au regard de la sensibilité de ces méthodes aux données d'entrée comme la configuration topo-bathymétrique, la reproduction des éléments urbains ou les scénarios de forçages.

\section{Site d'étude}

Le littoral étudié est le lido de la lagune Salse-Leucate, localisée à l'ouest du golfe du Lion (cf. figure 1). Ce cordon sableux d'une topographie généralement basse, possède une largeur variable comprise entre $300 \mathrm{~m}$ et $1,5 \mathrm{~km}$. Il est constitué sur sa partie marine de plages sableuses à systèmes de barres tridimensionnelles alors que le rivage interne sur la lagune présente des pentes douces et de faibles profondeurs. Le lido est entrecoupé par 3 passes, artificialisées permettant la connexion entre l'étang et la mer. L'analyse des évènements historiques de tempêtes a permis de mettre en évidence une vingtaine d'évènements depuis 2007. Parmi ces évènements, la tempête de mars 2013 avec des houles supérieures à $6 \mathrm{~m}$ constitue un des évènements les plus marquants. Selon les statistiques ANEMOC, ces hauteurs correspondent à une période de retour de 30 à 50 ans. Le niveau d'eau maximum associé à cet évènement au marégraphe de Sète $(0,73 \mathrm{~m} \mathrm{NGF})$ a quant à lui une période de retour comprise entre 1 et 5 ans (CETMEF 2013). Cette tempête de très forte intensité, bien documentée tant du point de vue des conditions marines que des impacts sur le lido en matière de submersion marine, a servi référence et a permis de valider la mise en place des modèles. 


\section{XVèmes Journées Nationales Génie Côtier - Génie Civil \\ La Rochelle, 29 au 31 mai 2018}
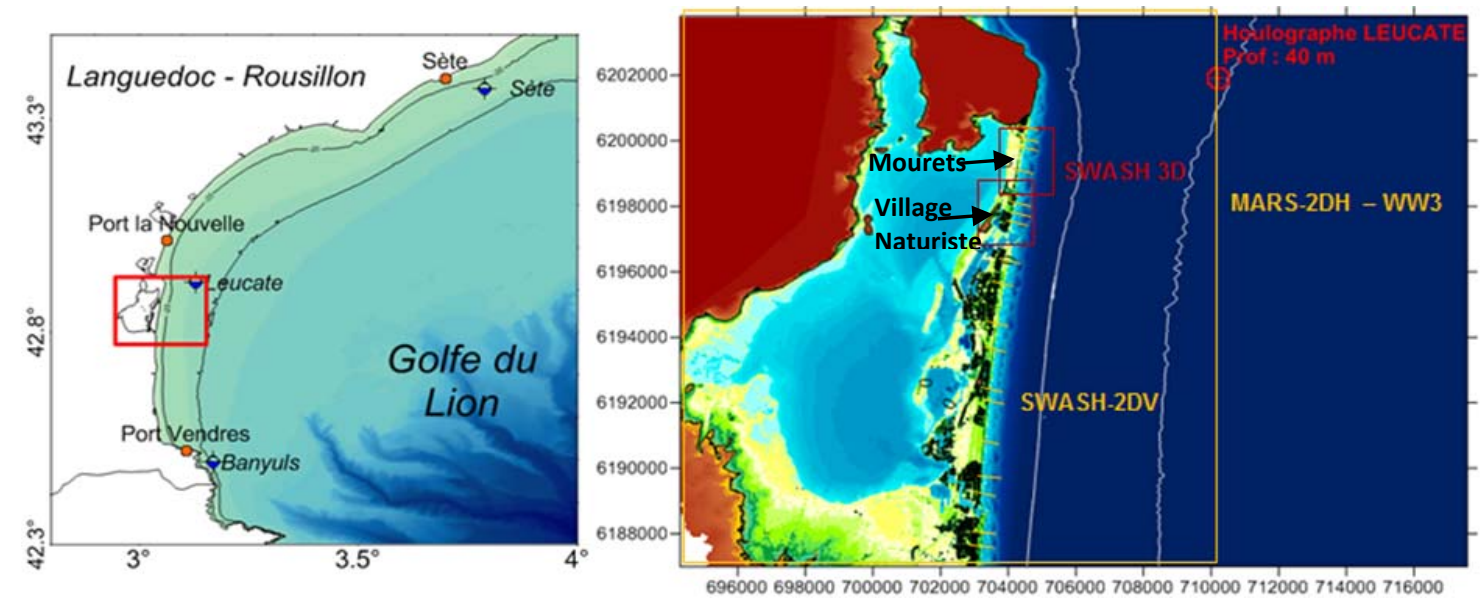

Figure 1. Localisation du site d'étude et extension des domaines de modélisation, à gauche la position des marégraphes (points oranges) et des houlographes (en bleu).

\section{Méthode}

Simuler les volumes franchissants exige de reproduire l'évolution des niveaux d'eau statiques sur la plage ainsi que la propagation des conditions de vagues du large à la côte. Pour se faire, un couplage du code spectral de vagues WW3 (TOLMAN 2009) avec un code hydrodynamique de niveaux et courants MARS-2DH (LAZURE \& DUMAS, 2008) a été réalisé sur un domaine couvrant l'ensemble de la lagune (résolution $10 \mathrm{~m}$ ). Pour reproduire les submersions de franchissement par paquets de mer, plusieurs types de modèle hydrodynamique peuvent être utilisés (cf. NICOLAE LERMA et al., 2018). Le modèle mis en place ici est le modèle SWASH (ZIJLEMA et al., 2011), modèle de type Non Linear Shallow Water dit à résolution de phase. Ce type de modèle permet de reproduire la dynamique du niveau d'eau instantané en résolvant l'hydrodynamique liée aux vagues (évolution du plan d'eau, calculs des courants engendrés par les vagues, interactions vague-vague et vague-courant, écoulements à terre) dans la zone littorale, la zone de déferlement et à terre en cas de submersion. Le modèle SWASH est chainé aux sorties de la plateforme de modélisation hydrodynamique (niveaux/vagues). Les forçages sont réalisés hors de la zone de déferlement afin que l'ensemble des processus de variation du niveau d'eau liés au déferlement (wave setup et runup) soit assuré par le modèle SWASH.

Contrairement aux modèles utilisés pour déterminer les hauteurs d'eau et vagues à la côte qui permettent une relative souplesse en termes de temps de calcul et de résolution spatiale de travail, la modélisation des franchissements exige à l'inverse des ressources et des temps de calculs prohibitifs dès lors que les domaines dépassent quelques kilomètres carrés. Ainsi, deux stratégies sont mises en place pour l'évaluation des franchissements (cf. figure 2). La stratégie 1 permet d'évaluer l'aléa à l'échelle de l'ensemble du site via des simulations pseudo 2DV (dimension cross shore et 2 couches verticales) sur des profils de plage. Chaque profil est défini comme représentatif d'un 


\section{Thème 7 - Risques côtiers}

tronçon de littoral pour lequel les caractéristiques morphologiques et d'exposition aux vagues sont considérées comme homogènes. Une fois les simulations du déferlement des vagues réalisées sur les profils, une nouvelle simulation est effectuée : les valeurs de volumes franchissant le front de mer sont réinjectées au sein du modèle hydrodynamique configuré pour reproduire les écoulements à terre en fonction des caractéristiques d'occupation du sol.

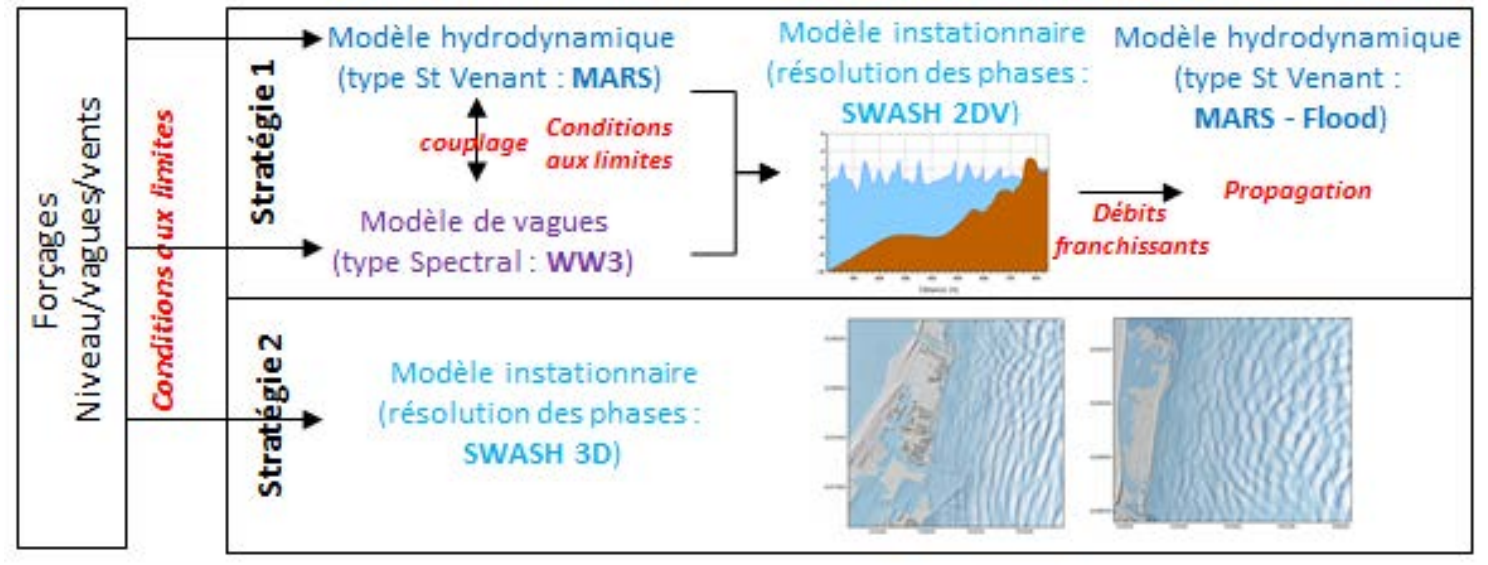

Figure 2. Illustration des stratégies de modélisation des franchissements.

La stratégie 2 est déployée à l'échelle locale des simulations pseudo 3D (2 couches verticales) sur un domaine de $2 \times 2 \mathrm{~km}$ d'extension, de résolution spatiale $2 \mathrm{~m}$.

Enfin, afin de réaliser des modèles numériques adaptés à la simulation numérique de la submersion, de nombreux traitements ont été réalisés (élaboration de coefficient de rugosité variable spatialement et en fonction de la représentation du bâti, lissage de rugosités artificielles, rehaussement des lignes de contrainte ...)

\section{Résultats}

\subsection{Validation des modélisations pour l'évènement historique}

La comparaison des résultats de modélisation et des documents disponibles (photos et films) montrent que la stratégie 2 semble reproduire de manière fidèle les submersions tant du point de vue de l'extension des zones inondées que des hauteurs d'eau (figure 3). En effet, des photos et des vidéos prises durant l'évènement, et qui permettent d'apprécier au moins qualitativement la conformité des résultats de simulation avec les témoignages disponibles, suggèrent que les hauteurs d'eau dans les rues ont été relativement faibles $(10$ à $20 \mathrm{~cm}$ ) ce qui est correctement reproduit par le modèle. En revanche, la stratégie 1 ne permet pas de reproduire les submersions, aucun franchissement n'a eu lieu pour les conditions de la tempête 2013. 


\section{XVèmes Journées Nationales Génie Côtier - Génie Civil La Rochelle, 29 au 31 mai 2018}
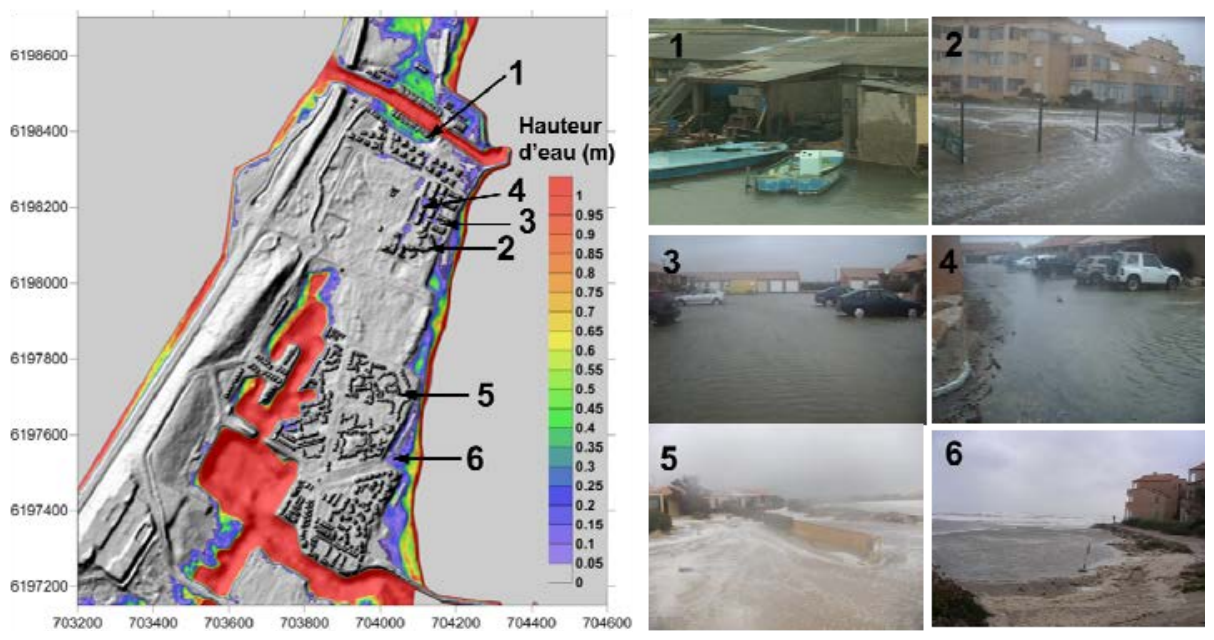

Figure 3. Validation des simulations de la tempête de mars 2013.

\subsection{Comparaison des stratégies pour un évènement centennal}

Ici le scénario retenu comme hypothèse de travail est le scénario centennal issu d'une analyse en probabilité conjointe proposée dans NICOLAE LERMA et al. (2018).

Pour le site du village naturiste la simulation de l'évènement centennal donne des résultats comparables pour les deux stratégies de modélisation. En revanche, sur le site des Mourets (cf. figure 1), des différences notables apparaissent en fonction de la stratégie retenue (cf. figure 4).

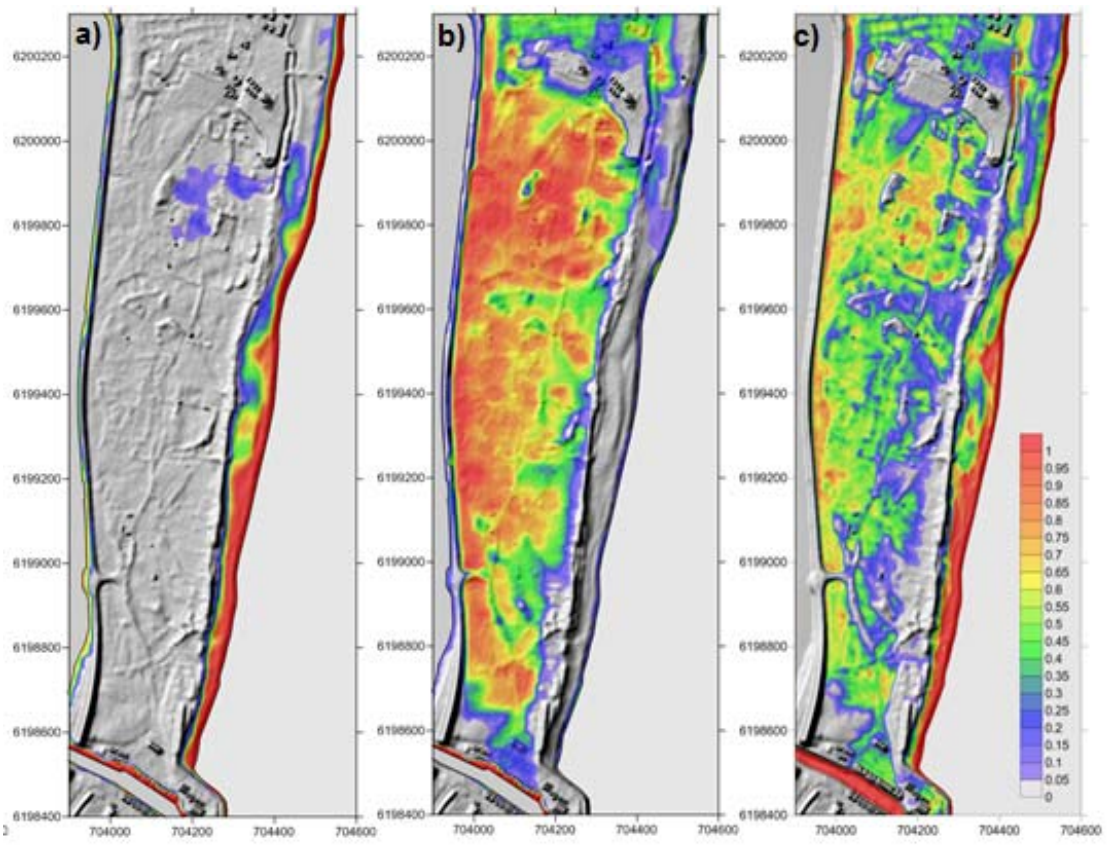

Figure 4. Résultats des simulations pour un évènement centennal, a) uniquement processus de débordement, b) stratégie 1, c) stratégie 2. 


\section{Thème 7 - Risques côtiers}

Sur ce site, comme pour le village naturiste, la submersion pour un évènement centennal est quasi exclusivement causée par des franchissements (cf. figure 4a).

La comparaison des deux stratégies présente des différences importantes en termes de hauteurs d'eau. En effet, la stratégie 1 propose une estimation des hauteurs d'eau très nettement supérieure du fait de volumes franchissants nettement plus importants. L'effet de la résolution spatiale sur la représentation de l'aléa est également visible ici, la propagation des volumes franchissants à une résolution de $10 \mathrm{~m}$ issue de la stratégie $1 \mathrm{a}$ tendance à lisser la délimitation des zones inondées, alors que les différentiels de hauteur d'eau sont nettement marqués pour la stratégie 2 (résolution spatiale $2 \mathrm{~m}$ ).

\subsection{Morphologie des profils et volumes franchissants}

Une des limites inhérentes aux méthodes mise en place est l'utilisation d'un modèle topo-bathymétrique à fond fixe. Cette méthode implique que la morphologie de la bande littorale et de la plage n'évolue pas en fonction des conditions hydrodynamiques. En outre, elle est représentative de l'état du système au moment où les levés ont eu lieu, or ceux-ci sont généralement réalisés par temps calme et rarement en hiver. La plage présente donc une configuration morphologique représentative d'un moment précis lors de conditions favorables.

Afin d'évaluer l'impact de la morphologie sur les volumes pouvant potentiellement franchir le front de mer, des tests d'évaluation de l'influence de la morphologie de la plage sous-marine, de l'estran et du haut de plage ont été réalisés. Pour cela, un profil type caractéristique de la zone du front de mer au nord du village naturiste a été sélectionné et 5 configurations morphologiques du profil de plage ont été testées (figure 5). Les conditions de forçages utilisées sont présentées dans le Tableau 1.

La morphologie du profil joue un rôle important dans les volumes franchissants en fonction de l'intensité de la tempête. Plus l'évènement est intense plus la morphologie $\mathrm{du}$ profil au moment de l'évènement jouera un rôle déterminant. Ainsi, pour une tempête très intense (de type "mars 2013") les tests montrent que seuls des changements très importants ou relatifs à des structures de protections en front de mer auront une incidence notable. En revanche, lors d'évènements exceptionnels (période de retour 100 ans), un changement de morphologie classique entre un profil d'hiver et un profil d'été ou associé à la variabilité longshore de la position des barres sous-marines peut provoquer la multiplication par trois des volumes franchissants totaux.

Tableau 1. Conditions de forçages utilisées pour les tests de sensibilité.

\begin{tabular}{llll}
\hline $\begin{array}{l}\text { Type d'évènement } \\
\text { (cas) }\end{array}$ & Niveau d'eau (m NGF) & $\begin{array}{l}\text { Hs } \\
(\boldsymbol{m})\end{array}$ & $\begin{array}{l}\text { Tp } \\
\text { (s) }\end{array}$ \\
\hline Tempête classique & & 5.0 & 10.0 \\
Tempête type mars 2013 & 0.80 & 6.2 & 11.0 \\
Tempête centennale & 0.92 & 6.7 & 11.2 \\
\hline
\end{tabular}




\section{XVèmes Journées Nationales Génie Côtier - Génie Civil \\ La Rochelle, 29 au 31 mai 2018}

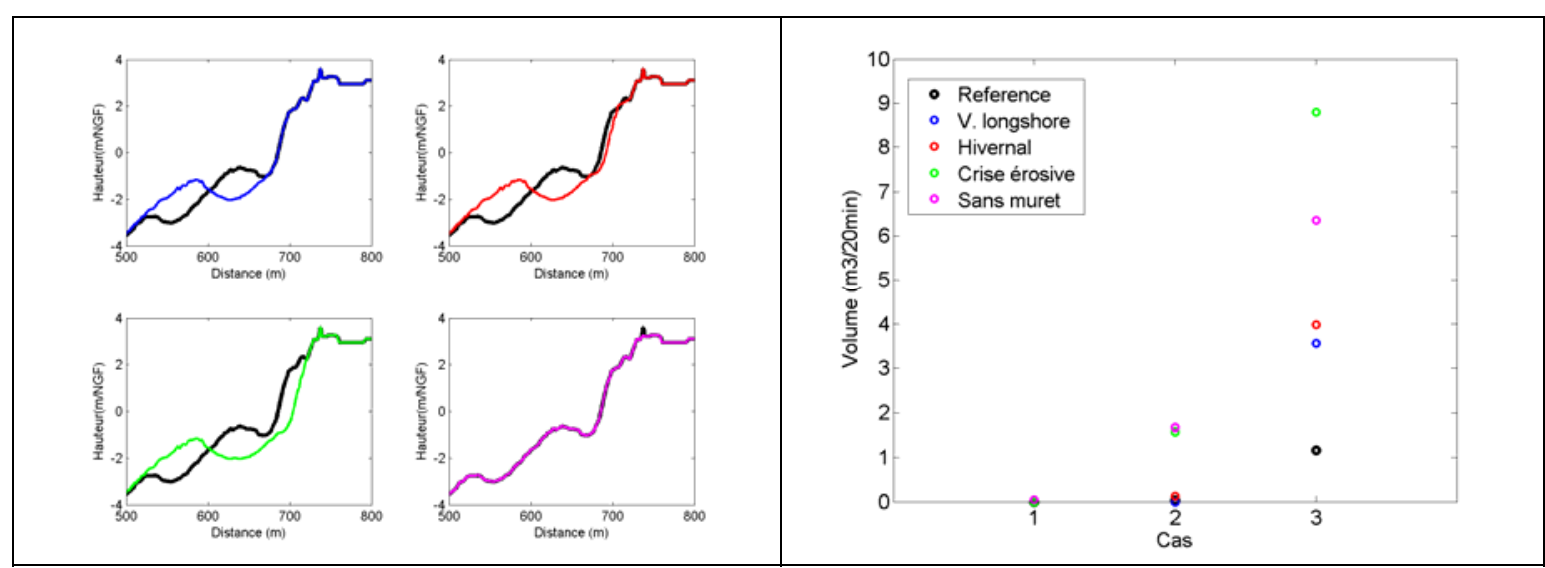

Figure 5. A gauche, profils de plage utilisés pour la réalisation des tests de sensibilité.

Profil de reference (noir), profil type "variabilité longshore" (bleu), profil de type "hivernal" (rouge), profil de type "crise érosive" (vert), profil "sans muret" (magenta). A droite, volumes franchissants en fonction de la morphologie du profil de plage et du cas de tempête.

\section{Conclusion}

On retirera de ce travail que la stratégie 2 permet de s'affranchir de plusieurs contraintes inhérentes à la stratégie 1 (choix de la localisation des profils, localisation de l'introduction des volumes franchissants dans le modèle de submersion...). La stratégie $2 \mathrm{~s}$ 'avère ainsi particulièrement adaptée pour les sites urbains exposés comme le village naturiste. Pour un évènement de forte intensité comme la tempête de mars 2013, l'approche par profils (stratégie 1) a tendance à minimiser l'extension de l'aléa. Pour un évènement extrême, les résultats sont assez comparables même si les niveaux d'eau obtenus pour la stratégie 2 sont légèrement supérieurs à ceux de la stratégie 1 . En revanche, pour les sites moins densément urbanisés, et en l'occurrence ici où le cordon dunaire est de relativement faible hauteur (cf. site des Mourets où la hauteur est comprise entre 2,5 et $4 \mathrm{~m} \mathrm{NGF}$ ), la stratégie 1 a tendance à proposer des résultats maximisants par rapport à la stratégie 2. Ces différences entre stratégie 1 et 2 peuvent être dues à la représentativité des profils dont les variations morphologiques ont une incidence importante sur les volumes franchissants. Il convient donc d'analyser l'influence des variations morphologiques sur le volume franchissant au sein d'un même tronçon en cas d'application de la stratégie 1 .

Le passage de la stratégie 1 à 2 permet d'affiner la connaissance à l'échelle locale. La stratégie 2 permet en effet de décrire de manière intégrée l'ensemble des processus à l'origine des submersions (marée, surcote atmosphérique, contribution des vagues : ondes infra gravitaires et gravitaires). Elle permet ainsi de déterminer la variabilité longshore des franchissements, les points de fragilité et le comportement et les vitesses des écoulements en milieu urbain. Toutefois, il convient de rappeler que cette stratégie 


\section{Thème 7 - Risques côtiers}

est très couteuse en temps de mise en place et de calcul et qu'il reste nécessaire de valider les performances de ce type d'approche par des protocoles d'observations de terrain adaptés.

\section{Remerciements}

Cette étude a bénéficié d'un financement de la DREAL Occitanie et du BRGM. Les données de vague, niveaux d'eau (réseaux Candhis et Refmar) et LiDAR ont été fournies par la DREAL Occitanie.

\section{Références bibliographiques}

GALLIEN T.W. (2016) Validated coastal flood modeling at Imperial Beach, California: Comparing total water level, empirical and numerical overtopping methodologies. Coastal Engineering, Vol. 111, pp 95-104. https://doi.org/10.1016/j.coastaleng.2016.01.014

LAZURE P., DUMAS F. (2008). An external-internal mode coupling for a 3D hydrodynamical model for applications at regional scale (MARS). Advances in Water Resources, Vol. 31, pp 233-250. https://doi.org/10.1016/j.advwatres.2007.06.010

LECACHEUX S., PEDREROS R., NICOLAE LERMA A., CHATEAUMINOIS E., ROHMER J., BONNARDOT F., SAUVAGNARGUES S. (2016). An experimental cyclone-induced marine and river floods forecasting system for La Reunion Island: the SPICy project. European Conference on Flood Risk Management (FLOODrisk 2016).

LE ROY S., PEDREROS R., ANDRE C., PARIS F., LECACHEUX S., MARCHE F., VINCHON C. (2015). Coastal flooding of urban areas by overtopping: dynamic modelling application to the Johanna storm (2008) in Gâvres (France). Natural Hazards and Earth System Sciences, Vol. 15, pp 2497-2510. https://doi.org/10.5194/nhess-15$\underline{2497-2015}$

NICOLAE LERMA A., THOMAS Y.F., DURAND P., ANSELME B., ANDRADE C. (2013). Modélisation de submersions marines et leur validation par observation vidéo Carthagène des Indes, Colombie. Géomorphologie: relief, processus, environnement, Vol. 4, pp 481-498. https://doi.org/10.4000/geomorphologie.10417

NICOLAE LERMA A., BULTEAU T., ElineAU S., PARIS F., DURAND P., ANSELME B., PEDREROS R. (2018). High-resolution marine flood modelling coupling overflow and overtopping processes: framing the hazard based on historical and statistical approaches. Natural Hazards and Earth System Sciences, Vol. 18(1), pp 207-229. https://doi.org/10.5194/nhess-18-207-2018

TOLMAN H. L. (2009). User manual and system documentation of WAVEWATCH III TM version 3.14. Technical note, MMAB Contribution, 276, 220.

ZIJLEMA M., STELLING G.S., SMIT P.B. (2011). SWASH: an operational public domain code for simulating wave fields and rapidly varied flows in coastal waters, Coastal Engineering, Vol. 58, pp 992-1012. https://doi.org/10.1016/j.coastaleng.2011.05.015 